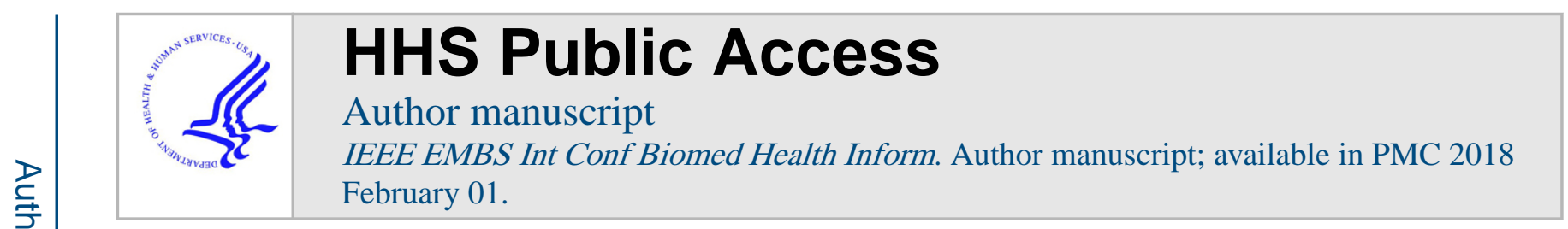

Published in final edited form as:

IEEE EMBS Int Conf Biomed Health Inform. 2017 February ; 2017: 181-184. doi:10.1109/BHI. 2017.7897235 .

\title{
Intelligent Mortality Reporting with FHIR
}

\author{
Ryan A. Hoffman, \\ Department of Biomedical Engineering, Georgia Institute of Technology and Emory University, \\ Atlanta, GA 30332 USA

\section{Hang Wu,} \\ School of Electrical and Computer Engineering, Georgia Institute of Technology, Atlanta, GA \\ 30332 USA
}

Janani Venugopalan, Department of Biomedical Engineering, Georgia Institute of Technology and Emory University, Atlanta, GA 30332 USA

Paula Braun, and Centers for Disease Control and Prevention (CDC), Atlanta, GA 30329 USA

\section{May D. Wang}

Department of Biomedical Engineering, Georgia Institute of Technology and Emory University, Atlanta, GA 30332 USA

\section{Abstract}

One pressing need in the area of public health is timely, accurate, and complete reporting of deaths and the conditions leading up to them. Fast Healthcare Interoperability Resources (FHIR) is a new HL7 interoperability standard for electronic health record (EHR), while Sustainable Medical Applications and Reusable Technologies (SMART)-on-FHIR enables third-party app development that can work "out of the box". This research demonstrates the feasibility of developing SMARTon-FHIR applications to enable medical professionals to perform timely and accurate death reporting within multiple different jurisdictions of US. We explored how the information on a standard certificate of death can be mapped to resources defined in the FHIR standard (DSTU2). We also demonstrated analytics for potentially improving the accuracy and completeness of mortality reporting data.

\section{Introduction}

Mortality is one of the most reliable sources of health-related data that is comparable across different geographical locations and is a large source of population-level health data, with approximately 56 million deaths per year world-wide [1]. In the United States of America (US) alone 2.6 million people die each year [2], with $60-80 \%$ being certified by physicians. Timely and accurate mortality data is essential for formulating emergency response to 
epidemics and new disease threats, prevention of communicable diseases such as flu, determining vital statistics such as life expectancy, mortality trends, etc.

Accurate collection and aggregation of high-quality mortality data remains an ongoing challenge primarily due to issues such as the lack of practice for physicians to perform death certification (on the order of 1-2 times a year), non-standard methods to determine the cause of death information, complex data flow between the funeral home, the certifying physician and the registrar, non-standard practices of data acquisition and transmission [3, 4]. The issue is further compounded by the fact that each location has different laws regarding the format of the death certificates and the type of information collected. For example, in the US, the National Center for Health Statistics aggregates mortality data from the 57 reporting jurisdictions around the country. However, the precise regulations and local laws of each reporting jurisdiction differ [5].

A decision support system that can assist the physician to fill the appropriate cause of death and put on the death certificate in the requisite format can largely assist to mitigate these challenges of data accuracy. Additionally, current efforts towards mortality reporting standardization using technologies such as HL7 V2 [6, 7] and CDA [8] have some shortcomings, such as challenges in integrating with large-scale web services. As a result, the current flow of information between the various providers and registrars is not optimal. Also, under the new meaningful use of EHR, the government requires healthcare institutions to show at least partial support of APIs to show potential for sharing data, interoperability and clinical decision support [9]. The current systems of HL7 and CDA do not have the capability to support APIs and incorporate decision support systems that facilitate the use of data from the decedent's electronic health records to notify the certifying medical professional of events that may be associated with the death.

To overcome these challenges, we propose a framework that utilizes HL7's Fast Healthcare Interoperability Resources (FHIR) as both an application platform and a means of accessing EHR data. FHIR is a new emerging health standard that is aimed at streamlining and standardizing healthcare communication using a resource-centric approach (as opposed to document-centric) for specification of data elements. It is designed to allow simple implementation using existing technologies such as restful APIs, OAuth security, and XML/ JSON data. [10]. FHIR was chosen for the application of death certificates, because it is vendor-neutral, scalable, and is positioned to emerge as a global standard. FHIR is designed to work within current EHR systems using APIs and can be used to pre-populate death certificates to aid physicians in determining the cause of death information. FHIR is also currently the only standard which supports the addition of analytics into the EHR systems $[11,12]$. The ultimate goals of this project are to generate information that will aid in more complete physician reporting of the causes of death, and to provide valuable mortality information to registrars, public health department, and other authorized parties in a timelier manner. 


\section{Web Application Design}

A web application was implemented in HTML and JavaScript, using the SMART-on-FHIR JavaScript client library (https://github.com/smart-on-fhir/client-js). The application runs in the browser, securely accessing the FHIR server using OAuth2 authentication. The application was developed and tested using a virtual FHIR server. The development server is based on a Vagrant VM configuration created by the SMART project team (https:// github.com/smart-on-fhir/installer). In addition to the SMART-on-FHIR compliant EHR server, an application server hosts CGI interfaces to UMLS and data mining functionality. An outline of the proposed infrastructure for a SMART-on-FHIR-based mortality is shown in Figure 1.

\section{A. Application Features}

This application was designed with the ultimate goal of enabling not just more timely, but also more accurate and complete data about the chain of events ultimately leading to death. As such, it is designed to allow the simultaneous visualization of a large portion of the patient history. As mortality reporting in the United States has adopted the ICD-10 standard since 1999, integration with the Unified Medical Language System (UMLS) Metathesaurus is necessary to enable crosswalk between medical event coding systems.

\section{B. Illustrative Synthetic Data}

To aid in interface prototyping and illustration of the application interface in Figure 2, synthetic patient data was created for patients with a variety of conditions surrounding a hypothetical recorded death.

\section{Interface Design}

The application main interface is illustrated in Figure 2. The application's interface is broken into horizontal panes. The topmost panes recapitulate the patient's information, displaying basic identification data as well as recent noted entered into the patient's record. This information is pulled from Patient, Condition, and other FHIR resources.

The largest, central pane displays an interactive patient history timeline generated using the popular D3 and D3-tip visualization libraries (https://d3js.org, https://github.com/Caged/d3tip). Events displayed on the timeline are spaced logarithmically, with the axis anchored at the time of death. This allows simultaneous visualization of events occurring around the time of death alongside relevant context from the patient's more distant history. Scrolling adjusts the scaling to allow focus on past and recent events. The events shown on this timeline are generated using Condition resources accessed from the FHIR server.

The bottommost panes are designed so as to recreate the familiar-to-users appearance of the US Standard Certificate of Death's cause-of-death field layout, with a chain of one or more events occurring as consequences of one another.

Buttons are provided to access important features, such as closing the application, submitting a death certificate package, or interfacing with UMLS. 


\section{Sequential Pattern Mining Analytics}

This application illustrates how next-generation web services can be developed to aid in timely, accurate mortality reporting. To better understand the availability of this data and demonstrate this capability, we use sequential pattern mining on one year of NVSS data to mine a list of rules that can be used directly in the application to propose common pathways of events that may have lead to death.

\section{A. Data}

National Vital Statistics System, coordinated by the National Center for Health Statistics aggregates the causes of death for all deaths occurring within the United States from 1959 to 2014 [2]. Each death certificate format in vital statistics offices of each state, the District of Columbia, and other special jurisdictions varies, but generally consists of the underlying cause of death as recorded by physicians and other details such as the demographics, comorbid conditions, race and ethnicity.

\section{B. SPM Background and Related Work}

The temporal models commonly seen in the literature include models such as sequence analysis [13-17] and association rule mining [16, 18, 19]. Sequential Pattern Mining (SPM) is a data mining technique that seeks temporal relationships among events (in this case the underlying causes of death) [20] and has been extensively examined in the literature with applications in pattern mining [21] (AprioriAll [22], SPADE [23]) and database projections. (PrefixSpan [24], MEMISP [25]). Recently, privacy preserving pattern mining [26-28] and distributed mining [26] have attracted considerable interests. In health care, SPM has applications in heart disease prediction [29], healthcare auditing [30], and neurological diagnosis [31], violent death reporting [32] etc. The input data to an SPM is a set of sequences, which comprises a list of events ordered by temporal relations.

The goal of SPM is to discover all valid sequential patterns with pre-specified minimum support, where support of a candidate pattern is the proportion of sequences in the data that exhibit the pattern [33]. For example, in the Multiple Cause-of-Death data, each record contains a list of ordered conditions (up to 20 conditions) that could lead to a person's death. While SPM's output an ordered list of sequences which correlates with the target outcome and is able to find rules such as "Condition $1->$ Condition 2". This means that if we observe Condition 1, we can assert that Condition 2 will possibly follow Condition 1. This was introduced as an improvement over Association Rule Mining (ARM) [21], which doesn't take the temporal relations into consideration, so will only output rules like "[Condition 1, Condition 2]", meaning if we observe Condition 1, we are also likely to observe Condition 2 for some confidence. Hence SPMs form an ideal algorithm for the current task of discovering the most probable sequence of events that led to the cause of death to help certifying physicians fill the death certificates.

\section{SPM Problem Formulation}

As discussed above, the NCHS database consists of up to 20 underlying conditions $C=\left[C_{1}\right.$, $\left.C_{2}, \ldots, C_{K}\right]$ which lead to death, where $C=\left\{C_{1}, C_{2}, \ldots, C_{K}\right\}$ is the list of unique events/ 
conditions. Using this data as the training, set our goal is find the list of most frequent sequence conditions $S=\left\langle s_{1}, s_{2}, \ldots, s_{T}\right\rangle$ which can occur given the outcome and comorbidities. A sequence $S$ is an ordered set of items, denoted as $S=\left\langle s_{1}, s_{2}, \ldots, s_{T}\right\rangle$, where each $e_{i} \in C$ is an item and the sequence is of length $T$. A set of sequences $D$ is a collection of sequences, $D=\left\{S^{(1)}, S^{(2)}, \ldots, S^{(N)}\right\}$, where the superscript denotes the index of an individual sequence and $N$ is the number of total number sequences.

We define the contain relationship $\subset$ between two sequences $A, B$ as follows: For $A=\left\langle a_{1}\right.$, $\left.a_{2}, \ldots, a_{T 1}\right\rangle, B=\left\langle b_{1}, b_{2}, \ldots, b_{T 2}\right\rangle$, if $\forall a_{i} \in A, \exists m(i)$, s.t. $b_{m(i)} \in B$, then $A \subset B$. A sequential pattern, or a rule, $R$ of a sequence $S$ is an ordered list that satisfies $R \subset S$. The relative sup- port of a rule $R$ in the set of sequences $D$ is defined as the percentage of sequences that contain this rule, i.e.,

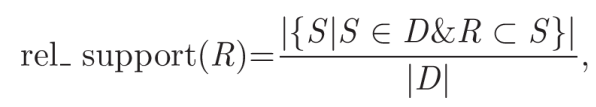

Where $\mid \cdot$ is the cardinality of a set. And we could also use the number of sequences that contain the rule as an evaluation metric, i.e., $\operatorname{support}(R)=|\{S \mid S \in D \& R \subset S\}|$.

Oftentimes, we want to find rules of length maximum possible, which leads to the definition of frequent closed rule. A rule $R$ is a frequent closed rule if there $\exists$ no $P$, s.t. $R \subset P$ and $\operatorname{support}(R)=\operatorname{support}(P)$.

SPM aims to discover sequential patterns that have support larger than a pre-specified minimum support. For example, we have the following set of sequences in Table 2 (the arrow "->" stands for temporal orders) and want to identify rules with a minimum support of 0.8 .

\section{SPM Methodology}

In our experiments, we use the BIDE algorithm, short for BI-Directional-Extension-based frequent closed sequence mining, proposed in Wang et al. [34]. Conventional sequence mining algorithms adopt a candidate maintenance- and-test paradigm, in which they maintain a list of discovered closed rules and use the rules to prune the search space and determine whether new rules are promising to be closed. Such paradigm is accurate but lacks scalability with respect to the number of frequent closed rules, both in time and storage. On the other hand, the BIDE algorithm aims to find all the frequent closed rules, without candidate maintenance.

\section{E. Pattern Mining Results}

We apply the aforementioned algorithm BIDE to Multiple Cause-of-Death Mortality Data from NCHS to find most frequent sequences of conditions before people's deaths. We picked Year 2012's Mortality Data, which contains 2, 547, 864 deaths. We set the minimum support to be 50 and identified a total of 65,915 frequent closed rules. We present the distribution of rules of different lengths in Table 1. 
Due to the space limits, we present the top 5 rules of length- 2 in Table 2 for illustration. The full set of rules was deployed as a lookup table service using a CGI script, integrating it into the death reporting prototype application.

\section{Conclusions and Future Work}

This work demonstrates the feasibility of using the SMART-on-FHIR application framework to develop public health applications for mortality reporting, improving the timeliness and accessibility of such reports. Intelligent analytics have been show integrated with the prototype application, demonstrating future potential for improving the accuracy of death reporting. Future work may focus on using alternative data sets, as well as the more complete patient information exposed though the interoperability of FHIR, to construct more personalized and precise analytics systems. Further development is ongoing to develop precise FHIR resource profiles to concisely, completely, and flexibly represent death certificate data.

\section{Acknowledgments}

The authors thank Dr. Mark Braunstein (Georgia Tech), Dr. Myung Choi (Georgia Tech Research Institute), and Charles Sirc (CDC) for their invaluable assistance and support in shaping this project.

This work was supported in part by grants from the National Center for Advancing Translational Sciences of the National Institutes of Health (NIH) under Award UL1TR000454 to Dr. May D. Wang, T32 GM105490 Traineeship to Dr. Greg Gibson of Georgia Tech for trainee R. A. Hoffman, National Science Foundation Award NSF1651360, and the US Department of Health and Human Services (HHS) Centers for Disease Control and Prevention (CDC) HHSD2002015F62550B to Dr. May D. Wang, and Microsoft Research and Hewlett Packard. This article does not reflect the official policy or opinions of the CDC, NSF, or the US Department of HHS and does not constitute an endorsement of the individuals or their programs.

\section{References}

1. W. H. O. (W.H.O.). , editor. Top 10 Causes of Death - Factsheet. 2013.

2. N. C. f. H. Statistics. Health, United States, 2014: With special feature on adults aged 55-64. 2015

3. Randall B. Death Certification: A Primer Part I-An Introduction to the Death Certificate. South Dakota Medicine. 2014; 67

4. N. C. f. H. Statistics., editor. Possible Solutions to Common Problems in Death Certification. Centers for Disease Control and Prevention; 1997.

5. Cowper DC, Kubal JD, Maynard C, Hynes DM. A primer and comparative review of major US mortality databases. Annals of epidemiology. 2002; 12:462-468. [PubMed: 12377423]

6. Dolin RH, Alschuler L, Boyer S, Beebe C, Behlen FM, Biron PV, et al. HL7 clinical document architecture, release 2. Journal of the American Medical Informatics Association. 2006; 13:30-39. [PubMed: 16221939]

7. Dolin RH, Alschuler L, Beebe C, Biron PV, Boyer SL, Essin D, et al. The HL7 clinical document architecture. Journal of the American Medical Informatics Association. 2001; 8:552-569. [PubMed: 11687563]

8. Eichelberg, M., Aden, T., Riesmeier, J., Dogac, A., Laleci, GB. Electronic health record standards-a brief overview; Proceedings of the 4th IEEE International Conference on Information and Communications Technology (ICICT 2006); 2006.

9. Blumenthal D, Tavenner M. The "meaningful use" regulation for electronic health records. New England Journal of Medicine. 2010; 363:501-504. [PubMed: 20647183]

10. Bender, D., Sartipi, K. HL7 FHIR: An Agile and RESTful approach to healthcare information exchange; Computer-Based Medical Systems (CBMS), 2013 IEEE 26th International Symposium on; 2013. p. 326-331. 
11. Alterovitz G, Warner J, Zhang P, Chen Y, Ullman-Cullere M, Kreda D, et al. SMART on FHIR Genomics: Facilitating standardized clinicogenomic apps. Journal of the American Medical Informatics Association. 2015; 22:1173-1178. [PubMed: 26198304]

12. Smits M, Kramer E, Harthoorn M, Cornet R. A comparison of two Detailed Clinical Model representations: FHIR and CDA. European Journal for Biomedical Informatics. 2015; 11

13. Tao, C., Wongsuphasawat, K., Clark, K., Plaisant, C., Shneiderman, B., Chute, CG. Towards event sequence representation, reasoning and visualization for EHR data; presented at the Proceedings of the 2nd ACM SIGHIT International Health Informatics Symposium; Miami, Florida, USA. 2012;

14. Wang, TD., Plaisant, C., Quinn, AJ., Stanchak, R., Murphy, S., Shneiderman, B. Aligning temporal data by sentinel events: discovering patterns in electronic health records; Proceedings of the SIGCHI conference on Human factors in computing systems; 2008. p. 457-466.

15. Syed, H., Das, AK. Artificial Intelligence in Medicine. Springer; 2015. Identifying Chemotherapy Regimens in Electronic Health Record Data Using Interval-Encoded Sequence Alignment; p. 143-147.

16. Casanova, IJ., Campos, M., Juarez, JM., Fernandez-Fernandez-Arroyo, A., Lorente, JA. Using Multivariate Sequential Patterns to Improve Survival Prediction in Intensive Care Burn Unit. In: Holmes, HJ.Bellazzi, R.Sacchi, L., Peek, N., editors. Artificial Intelligence in Medicine: 15th Conference on Artificial Intelligence in Medicine, AIME 2015, Pavia, Italy, June 17-20, 2015. Proceedings. Cham: Springer International Publishing; 2015. p. 277-286.

17. Batal, I., Valizadegan, H., Cooper, GF., Hauskrecht, M. A pattern mining approach for classifying multivariate temporal data; Bioinformatics and Biomedicine (BIBM), 2011 IEEE International Conference on; 2011. p. 358-365.

18. Yang H, Yang CC. Using Health-Consumer-Contributed Data to Detect Adverse Drug Reactions by Association Mining with Temporal Analysis. ACM Trans. Intell. Syst. Technol. 2015; 6:1-27.

19. Bellazzi R, Ferrazzi F, Sacchi L. Predictive data mining in clinical medicine: a focus on selected methods and applications. Wiley Interdisciplinary Reviews: Data Mining and Knowledge Discovery. 2011; 1:416-430.

20. Zhao Q, Bhowmick SS. Sequential pattern mining: A survey. ITechnical Report CAIS Nayang Technological University Singapore. 2003:1-26.

21. Agrawal, R., Srikant, R. Fast algorithms for mining association rules; Proc. 20th int. conf. very large data bases, VLDB; 1994. p. 487-499.

22. Agrawal, R., Srikant, R. Mining sequential patterns; Data Engineering, 1995. Proceedings of the Eleventh International Conference on; 1995. p. 3-14.

23. Zaki MJ. SPADE: An efficient algorithm for mining frequent sequences. Machine learning. 2001; 42:31-60.

24. Pei J, Han J, Mortazavi-Asl B, Wang J, Pinto H, Chen Q, et al. Mining sequential patterns by pattern-growth: The prefixspan approach. Knowledge and Data Engineering, IEEE Transactions on. $2004 ; 16: 1424-1440$.

25. Lin, M-Y., Lee, S-Y. Data Warehousing and Knowledge Discovery. Springer; 2002. Fast discovery of sequential patterns by memory indexing; p. 150-160.

26. Mistry, BR., Desai, A. Privacy preserving heuristic approach for association rule mining in distributed database; Innovations in Information, Embedded and Communication Systems (ICIIECS), 2015 International Conference on; 2015. p. 1-7.

27. Fu, AW-C., Wong, RC-W., Wang, K. Privacy-preserving frequent pattern mining across private databases; Data Mining, Fifth IEEE International Conference on; 2005. p. 4

28. Sathiyapriya K, Sadasivam GS. A survey on privacy preserving association rule mining. International Journal of Data Mining \& Knowledge Management Process. 2013; 3:119.

29. Soni J, Ansari U, Sharma D, Soni S. Predictive data mining for medical diagnosis: An overview of heart disease prediction. International Journal of Computer Applications. 2011; 17:43-48.

30. Concaro S, Sacchi L, Cerra C, Fratino P, Bellazzi R. Mining health care administrative data with temporal association rules on hybrid events. Methods Inf Med. 2011; 50:166-79. [PubMed: 21170468] 
31. Dolce G, Quintieri M, Serra S, Lagani V, Pignolo L. Clinical signs and early prognosis in vegetative state: a decisional tree, data-mining study. Brain Injury. 2008; 22:617-623. [PubMed: 18568716]

32. McNally MR, Patton CL, Fremouw WJ. Mining for Murder-Suicide: An Approach to Identifying Cases of Murder-Suicide in the National Violent Death Reporting System Restricted Access Database. Journal of forensic sciences. 2015

33. Srikant, R., Agrawal, R. Mining sequential patterns: Generalizations and performance improvements. Springer; 1996.

34. Wang J, Han J, Li C. Frequent closed sequence mining without candidate maintenance. Knowledge and Data Engineering, IEEE Transactions on. 2007; 19:1042-1056. 

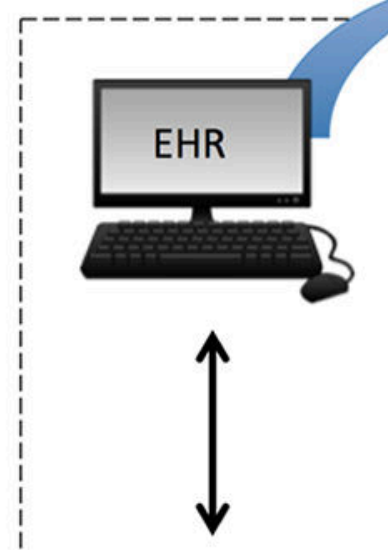

Figure 1.

\section{SMART-on-FHIR}

App Launch

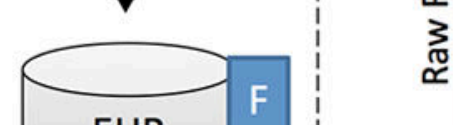

EHR

Back-End

Database

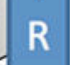

FHIR-Enabled

EHR Server

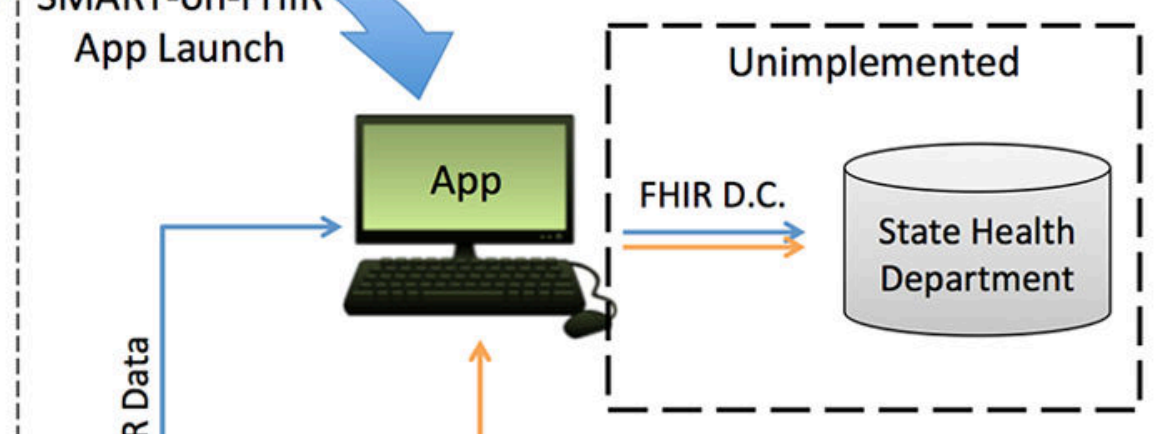

Advanced

Analytics

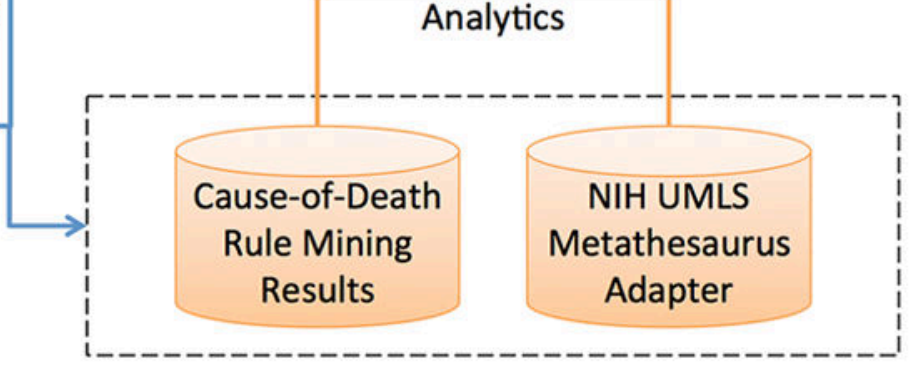

Application Server 
- D SMART FHIR Storter $\times \square$ GT/COC Death Roporting $\times \square \square$

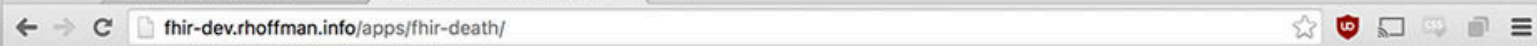

Johnston, Jonathan -- ID 110001

Patient Details

Name: Jonathan James Johnston

Age at death: 64.5 years

Residence: Everytown, USA 99999

Patient History

Lorem ipsum dolor sit amet, consectetur adipiscing elit. Suspendisse hendrerit, enim vel dictum dapibus, tellus massa dapibus nibh, in auctor felis felis ut mauris. Nam sit amet lorem diam. Sed ullamcorper magna eget enim semper, eu maximus nisi porta. Proin congue ex quam, ac rhoncus ipsum hendmerit anis Pmin sollicitudin diam vel diam semner ac norta felis convallis Nulla faucihus ricus

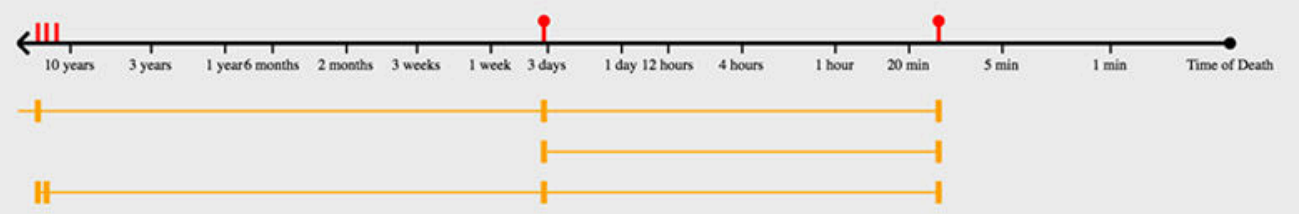

日 $\mathbf{\Psi}$

Cause of Death:

Rupture of cardiac wall without hemopericardium as current complication following acute myocarc

Acute myocardial infarction

ICD-10 Conversion
Onset to Death:

13 minutes

3 days
Other Significant Conditions

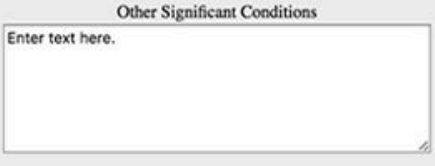

Figure 2. 
Table 1

\begin{tabular}{|c|c|}
\hline Length & Count \\
\hline 1 & 961 \\
\hline 2 & 19,160 \\
\hline 3 & 33,179 \\
\hline 4 & 11,508 \\
\hline 5 & 1,081 \\
\hline 6 & 26 \\
\hline$>7$ & 0 \\
\hline
\end{tabular}

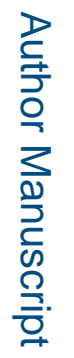

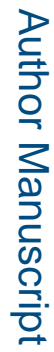

로을

IEEE EMBS Int Conf Biomed Health Inform. Author manuscript; available in PMC 2018 February 01. 


\section{Table 2}

\section{Rules from 2012 NCHS Mortality Data}

\begin{tabular}{|c|c|c|}
\hline Rule & Count & Percent \\
\hline Mental and behavioral disorders due to use of tobacco -> Other chronic obstructive pulmonary disease & 100,920 & $3.96 \%$ \\
\hline Chronic ischemic heart disease -> Cardiac arrest & 85,952 & $3.37 \%$ \\
\hline Essential (primary) hypertension -> Chronic ischemic heart disease & 77,249 & $3.03 \%$ \\
\hline Mental and behavioral disorders due to use of tobacco -> Malignant neoplasm of bronchus and lung & 73,212 & $2.87 \%$ \\
\hline Chronic ischemic heart disease -> Heart failure & 63,283 & $2.48 \%$ \\
\hline
\end{tabular}

